\title{
CLOSED LOOP CONTROL OF LASER WELDING USING AN OPTICAL SPECTROSCOPIC SENSOR FOR ND:YAG AND $\mathrm{CO}_{2}$ LASERS
}

Paper number 203

\author{
Ali Riza Konuk ${ }^{{ }^{1}}$, Ronald Aarts ${ }^{1}$, Bert Huis in 't Veld ${ }^{1}$, Teresa Sibillano $^{2}$, Domenico. Rizzi ${ }^{2}$, Antonio \\ Ancona ${ }^{2}$ \\ ${ }^{1}$ University of Twente, Laboratory of Mechanical Automation, \\ P.O. Box 217, 7500 AE Enschede, The Netherlands \\ ${ }^{2}$ CNR-IFN UOS Bari, Physics Department, via Amendola 173, I-70126 Bari, Italy
}

\begin{abstract}
Recent developments in laser joining show the applicability of spectral analysis of the plasma plume emission to monitor and control the quality of weld. The analysis of the complete spectra makes it possible to measure specific emission lines which reveal information about the welding process. The subsequent estimation of the electron temperature can be correlated with the quality of the corresponding weld seam. A typical quality parameter, for laser welds of stainless steel, is the achieved penetration depth of the weld. Furthermore adequate gas shielding of the welds has to be provided to avoid seam oxidation .

In this paper monitoring and real-time control of the penetration depth during laser welding is demonstrated. Optical emissions in the range of $400 \mathrm{~nm}$ and $560 \mathrm{~nm}$ are collected by a fast spectrometer. The sensor data are used to determine the weld quality of overlap welds in AISI 304 stainless steel sheets performed both with $\mathrm{CW}$ $\mathrm{Nd}$ :YAG and $\mathrm{CO}_{2}$ lasers. A PI-controller adjusts the laser power aiming at a constant penetration. Optical inspection of the weld surface and microscopic analysis of weld cross sections were used to verify the results obtained with the proposed closed-loop system of spectroscopic sensor and controller.
\end{abstract}

Keywords: Laser welding, Nd:YAG laser, plasma spectroscopy, melt pool control, electron temperature

\section{Introduction}

The information inside the plasma plume spectra of laser welding radiation is used to improve the weld quality or detect the flaws during welding process. [1] Analysis of the spectra is one of the techniques

\footnotetext{
*E-mail of corresponding author: a.r.konuk@ctw.utwente.nl
}

used to monitor and control the weld quality. [2] The real-time identification and classification of weld defects has proven to be non-trivial and usually it is performed by means of statistical studies of intensity of the emitted process light in one or more selected wavelength ranges $[3,4,5]$. Instead, the analysis of the complete spectra makes it possible to measure specific emission lines which reveal more information about the welding process. The consequent estimation of the electron temperature $T_{e}$ can be correlated with the quality of the corresponding weld seam. Sibillano et.al $[6,7]$ showed a clear relation between the estimated electron temperature and the penetration depth. In their work a $\mathrm{CO}_{2}$ laser has been used. In case of pulsed and continuous-wave $(\mathrm{CW}) \mathrm{Nd}: \mathrm{YAG}$ and $\mathrm{CO}_{2}$ welding electron temperature correlation is used for weld process penetration depth control. [8]

In this paper, a real-time PI controller is developed to stabilize the penetration depth of stainless steel welding process with overlapped welding configuration. The penetration depth is related with electron temperature calculated with $\mathrm{Cr}$ and $\mathrm{Fe}$ spectral line pairs for $\mathrm{Nd}: Y A G$ and $\mathrm{CO}_{2}$ laser welding respectively. Microscopic analysis of the cross sections was used to to check the penetration depths.

\section{Experimental Details}

Nd:YAG laser welding experiments were performed on AISI304 stainless steel sheets of $1 \mathrm{~mm}$ stacked up to $3 \mathrm{~mm}$ thickness. On the other hand, $\mathrm{CO}_{2}$ welding experiments were done in overlapped configuration of $1 \mathrm{~mm}$-thick sheet upon $2 \mathrm{~mm}$-thick AISI304 stainless steel plates. 
Figure 1 and Figure 2 illustrate schematically the two different welding system namely with $\mathrm{Nd}: \mathrm{YAG}$ and $\mathrm{CO}_{2}$ lasers. Overlapped welding experiments were conducted on the sheets. Nd:YAG (4 kW Trumpf THL4006D) and $\mathrm{CO}_{2}$ (Rofin Sinar DC025) laser sources in continuous wave $(\mathrm{CW})$ regime were used.

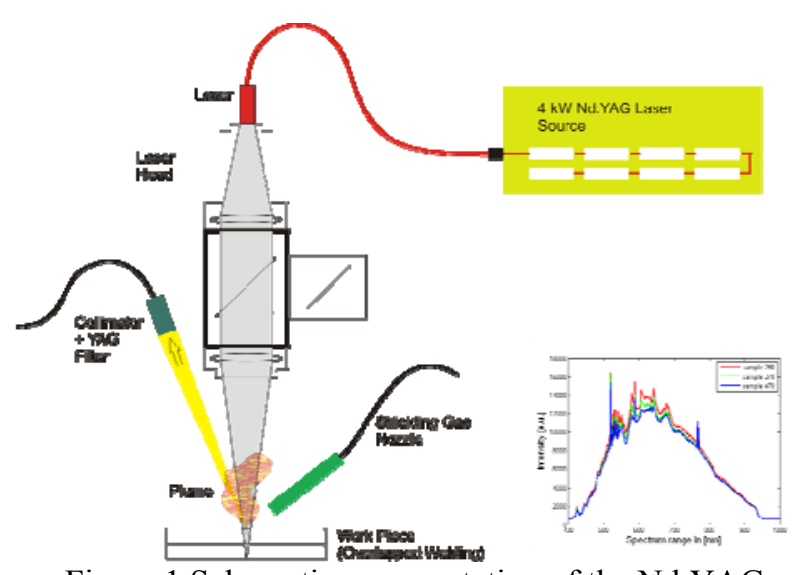

Figure 1 Schematic representation of the Nd:YAG experimental setup

A set of experiments was done using a Nd:YAG laser with a maximum power of $4 \mathrm{~kW}$ and beam quality of $25 \mathrm{~mm} \cdot \mathrm{mrad}$. The beam was guided through a $600 \mu \mathrm{m}$ fiber and was focused on the work-piece with a lens having a $200 \mathrm{~mm}$ focal length (Trumpf BEO70 head). Laser welding head was manipulated with a Stäubili RX-130 robot.

In parallel, another set of experiments with the $\mathrm{CO}_{2}$ laser was carried out using the maximum output power of $2.5 \mathrm{~kW}$.

An Ocean Optics spectrometer (HR2000+) was used to collect the optical plasma emission with a collimator for both systems. The quartz collimator had a focal length of $200 \mathrm{~mm}$ for Nd:YAG laser source. For $\mathrm{CO} 2$ welding, (Figure 2) the plasma optical emission was collected by a quartz collimator of $6 \mathrm{~mm}$ focal length and sent, through anoptical fiber with $200 \mu \mathrm{m}$ core-diameter, to a the spectrometer having a bandwidth of 390-525 nm and an optical resolution of $0.12 \mathrm{~nm}$. Nd:YAG collimator was focused onto the weld pool at an incident angle of $65^{\circ}$ as illustrated in figures 1 . An optical filter with a cut-off frequency of $900 \mathrm{~nm}$ was used to filter 1030 $\mathrm{nm}$ Nd:YAG laser radiations,. The collected light was transmitted to a calibrated linear CCD array (400-600 $\mathrm{nm}$ spectral range) by a quartz fiber.

A special tubular nozzle was used for shielding the weld melt pool from oxidation during the solidification process. Argon gas with a maximum flow rate of $1750 \mathrm{l} / \mathrm{h}$ was used for shielding during $\mathrm{Nd}$ :YAG welding. In $\mathrm{CO}_{2}$ laser welding the argon shielding gas flow rate was $60 \mathrm{l} / \mathrm{min}$ and the nozzle stand-off distance was $6 \mathrm{~mm}$.

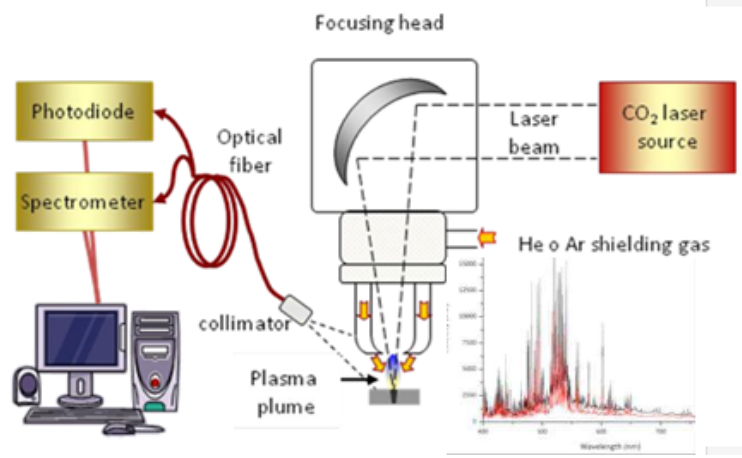

Figure 2 Schematic representation of the $\mathrm{CO}_{2}$ experimental setup

In optical data acquisition, the spectrometer was used at high speed acquisition mode. The sampling time including acquisition delay was found to be around 5 ms. This results in a maximum sampling rate of 200 $\mathrm{Hz}$. The integration time is adjusted in the range of $100-500 \mu \mathrm{s}$ according to the emission intensity to avoid saturation. The average to sample is set to 1 for $\mathrm{Nd}$ :YAG experiments. Average to sample indicates number of samples taken for averaging. In case of one there is not averaging performed. On the other hand, 10 samples are averaged in $\mathrm{CO}_{2}$ laser welding.

The signals received by the spectrometer is used to identify and control the process in Labview software platform for both $\mathrm{Nd}: \mathrm{YAG}$ and $\mathrm{CO}_{2}$ laser systems.

Two different type of welding experiments were made with the chosen stainless steel sheets. The welding speed was kept constant and the laser power was varied to characterize the weld process and for identification of the controller parameters. Based on this analysis, controller parameters are tuned for different laser sources used. Controlled welding experiments are made to find the penetration depth relation to electron temperature. In order to have a better sampling resolution along the weld length, the welding speed was adjusted to $60 \mathrm{~mm} / \mathrm{s}$ for Nd:YAG. In case of $\mathrm{CO}_{2}$ welding the welding speed was set to $50 \mathrm{~mm} / \mathrm{s}$. 


\section{Controller block diagram}

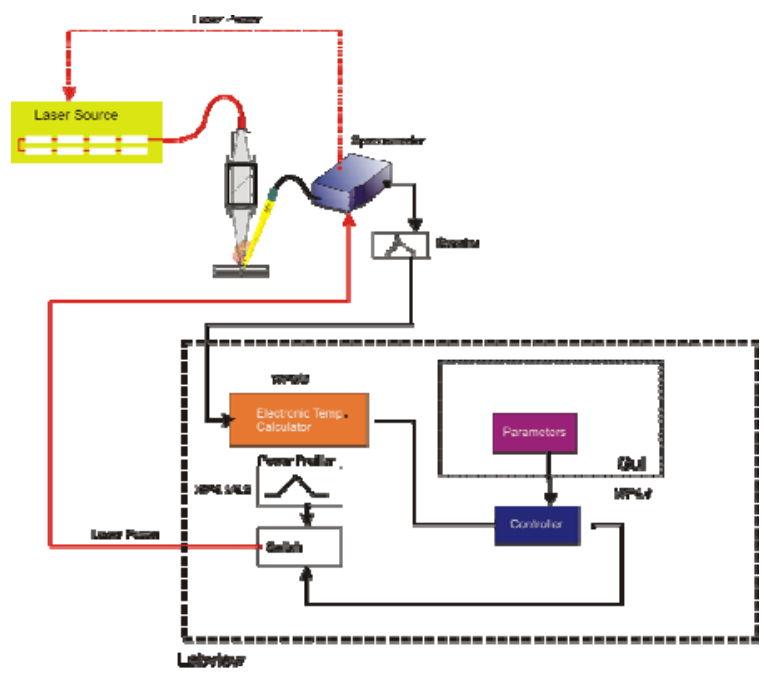

Figure 3 Block diagram of the controller using $\mathrm{HR} 2000+$ Spectrometer for $\mathrm{Nd}: \mathrm{YAG}$ and $\mathrm{CO}_{2}$ welding

Figure 3 shows an overview of the controller operation. The controller initially acquires the spectra from the spectrometer. The corresponding intensities at selected wavelengths are then used to calculate the electron temperature in the electron temperature calculator block. The temperature signal is then used by the controller to stabilize the penetration depth. Changing parameters like shielding gas flow or alignment of the collimator could affect the controller parameters. For that reason, a switch makes it possible to input a characterisation profile.

As a core, a PI controller (equation 1) is employed to control the penetration depth for different process conditions for $\mathrm{Nd}: \mathrm{YAG}$ and $\mathrm{CO}_{2}$ laser welding. In Figure 4 , the block diagram of the PI controller is shown. In the figure and equation $K_{p}$ and $T_{i}$ denote controller gain and integral time constant parameters, respectively. The process is shown as $P(s)$. The corresponding electron temperature set point for a desired penetration is set by adjusting the power output of the controller.

$$
C(s)=k_{p}\left(1+\frac{1}{T_{i} s}\right)
$$

Like the P-Only controller, the Proportional-Integral (PI) algorithm computes and transmits a controller output (CO) signal for every sample time, $T$, to the final control element (e.g., laser power, welding speed). The computed CO from the PI algorithm is influenced by the controller tuning parameters and the controller error, $\Delta T_{e}(t)$.

Integral action enables PI controllers to eliminate offset, which is not possible with a P-only controller. Thus, PI controllers provide a balance of complexity and capability that makes them a widely used algorithm in process control applications.

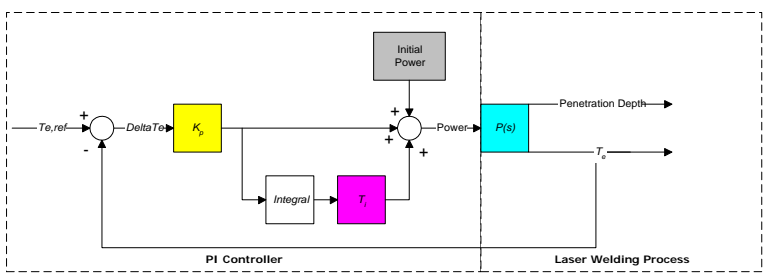

Figure 4 Block Diagram of the closed loop system with a PI controller

\section{Electron Temperature Calculation}

Significant peaks would give a reliable electron temperature $T e$ calculation based on Boltzmann plot method. This temperature $T e$ is calculated with the formula

$$
T_{e}=\frac{E_{m}(2)-E_{m}(1)}{k \ln \left(\frac{\mathrm{I}_{m n}(1) \lambda_{m n}(1) A_{m n}(2) g_{m}(2)}{\mathrm{I}_{m n}(2) \lambda_{m n}(2) A_{m n}(1) g_{m}(1)}\right)}
$$

where $I_{\mathrm{mn}}$ is the relative intensity of the emission line of the wavelength $\lambda_{\mathrm{mn}}(\mathrm{nm}), g_{m}$ is the statistical weight of the upper excited energy level with the energy $E_{m}(\mathrm{~cm}-1)$, and $A_{\mathrm{mn}}(\mathrm{s}-1)$ is the transition probability for spontaneous radiative emission taken from the NIST database. [9] This electron temperature calculation method gives the relation of the penetration depth using the right combination of atomic pair.

The atomic Chromium Cr I 459.23nm-495.69nm pair of lines is selected to calculate electron temperature during experiments with Nd:YAG laser. [10] Fe(I) couple: 421.91-517.09 $\mathrm{nm}$ is chosen for $\mathrm{CO}_{2}$ laser welding. [11] Based on the electron temperature calculation formula (Equation 2) the controller signal is calculated in real time during the welding process to stabilize the penetration depth

\section{Experimental Results and Discussion}

This section is divided into two parts showing the results of $\mathrm{Nd}$ :YAG and $\mathrm{CO}_{2}$ controlled welding separately. In both cases, materials characterisation analyses followed the controlled laser welding experiments.. 
The characterization experiments are necessary for each of the experiments. The shielding system changes or optical focus alterations can easily distort electron temperature value and will directly affect the desired penetration depth. For that reason, a proper characterization ensures a reliable relationship of electron temperature to penetration depth for the chosen configuration.

\section{Characterization of Nd:YAG Laser welding}

A V shape power profile starting at a high power of $3000 \mathrm{~W}$ and lowering to a minimum of $1400 \mathrm{~W}$ is used to identify the characteristics of the Nd:YAG welding process. In Figure 5, the green line shows the variation of the laser power. The blue line shows the observed electron temperature squeezed in the range 7600-7100 K.

In the same figure the penetration depth is depicted by bars with a bullet on each end. The height of each bar indicates the penetration measured from the transversal cross section. The value of the penetration depth is given as well on the upper side of the bar.

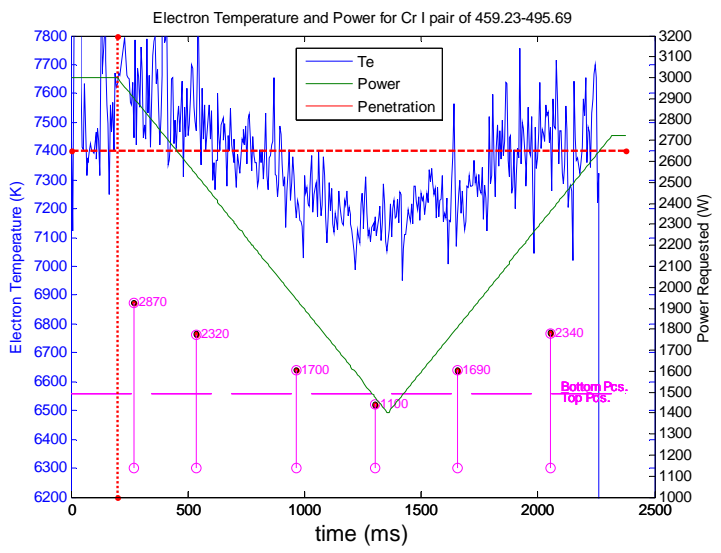

Figure 5 Characterisation of the Nd:YAG laser welding process with power variation between 1400 3000W

The optimum values of controller parameters are calculated based on this characterization plot. For the controlled experiments $K_{p}$ and $T_{i}$ values were set to 0.4 and $10 \mathrm{~ms}$, respectively.

As shown in the micrographs (Figure 7) of the cross sections, a low laser power of $1400 \mathrm{~W}$ corresponds to $7100-7200 \mathrm{~K}$ range of electron temperature which appears to cause only top plate penetration (Figure 7, lower left).

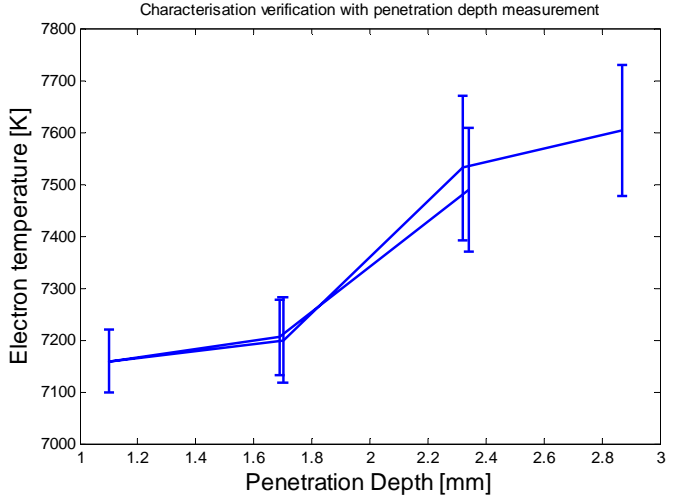

Figure 6 Electron temperature average and penetration relation based on the cross sections

To obtain a better penetration extending to the bottom plate, the set points were chosen to be $7300 \mathrm{~K}$ $7400 \mathrm{~K}$ and $7500 \mathrm{~K}$. Based on these values as shown in Figure 6, the expected penetration depths are $1.7 \mathrm{~mm}, 2.1 \mathrm{~mm}$, and $2.5 \mathrm{~mm}$ respectively.

Figure 7 clearly shows that laser power of $1400 \mathrm{~W}$ is sufficient to obtain full penetration $1 \mathrm{~mm}$ thickness of top plate. The large depth to width ratio of the weld zone in the six cross sections shown in this figure illustrate that keyhole was obtained during welding.
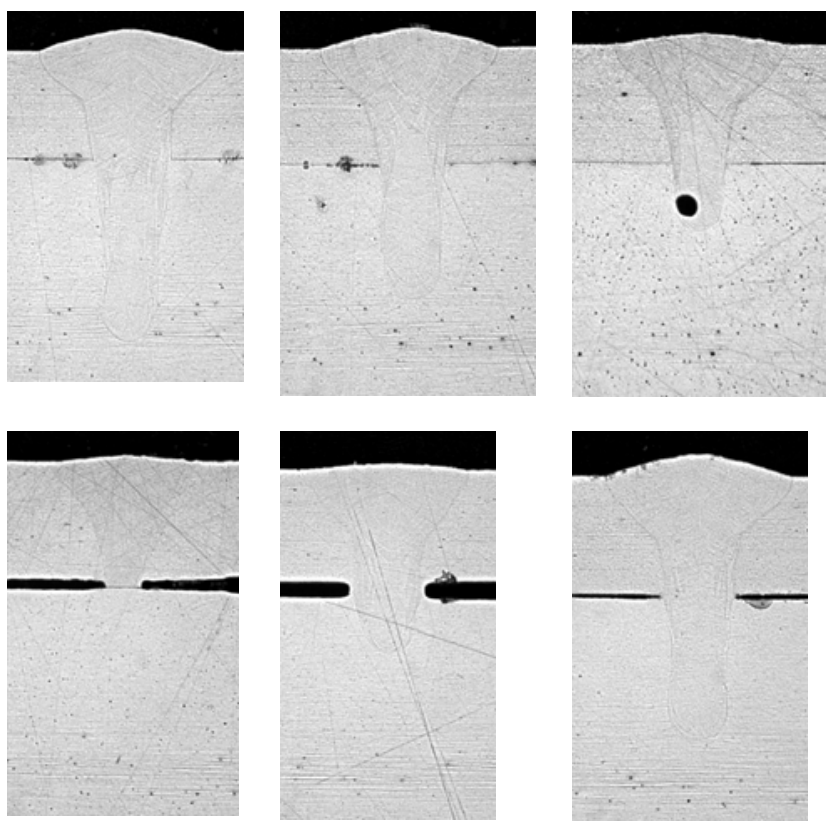

Figure 7 Six different transverse cross sections on same track of Nd:YAG laser welding with varied laser power. Welding speed is $60 \mathrm{~mm} / \mathrm{s}$, power variation is $1400 \mathrm{~W}-3000 \mathrm{~W}$ 


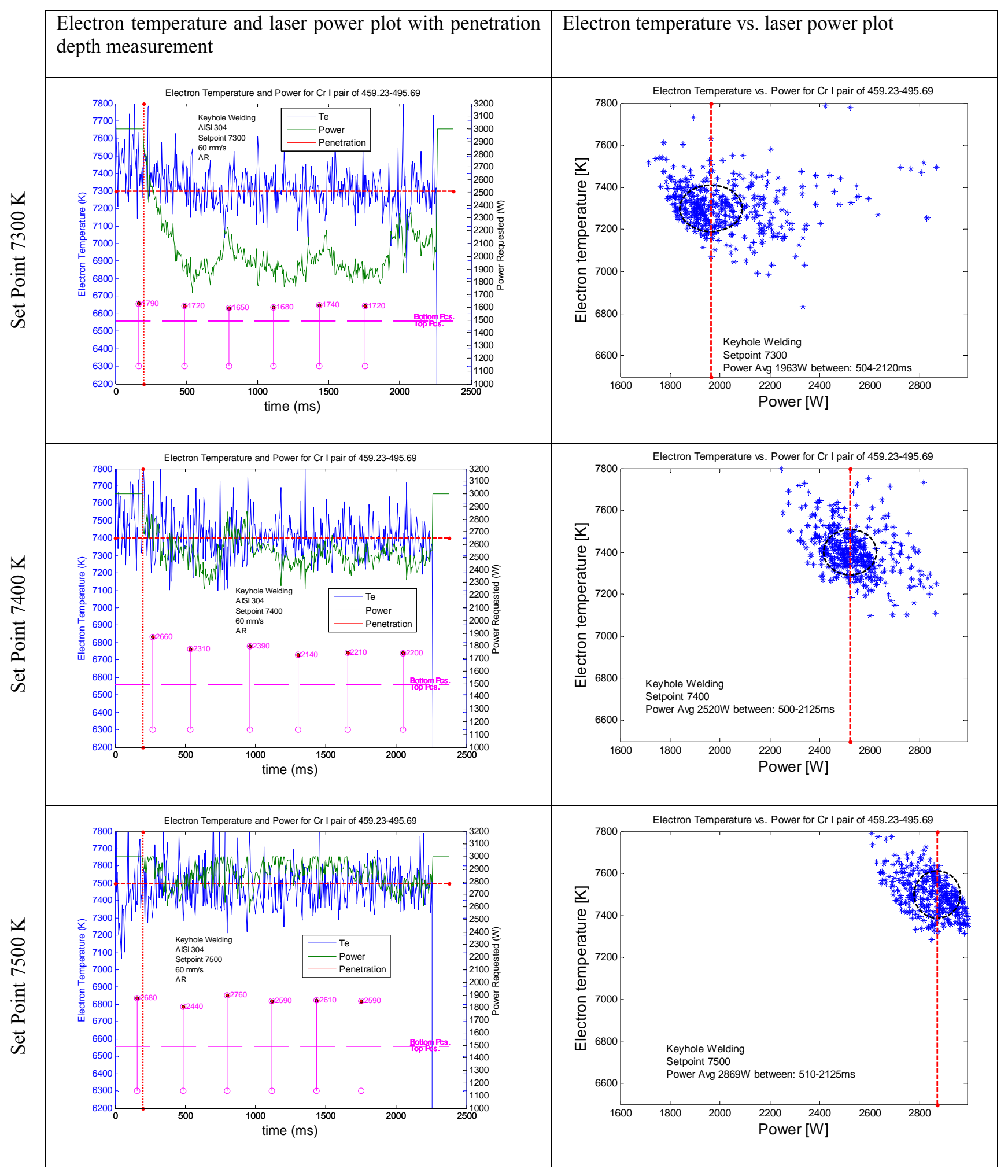

Figure 8 Electron temperature, power and penetration variations for $7300 \mathrm{~K}, 7400 \mathrm{~K}$, and $7500 \mathrm{~K}$ set points for Nd:YAG laser welding (left column). Electron temperature vs. Laser power relation of Nd:YAG welding (right column) 


\section{Controlled Experiments for Nd:YAG laser welding}

Three different set points of $7300 \mathrm{~K}, 7400 \mathrm{~K}$ and $7500 \mathrm{~K}$ are used as penetration depth controller. The controller is set off for $200 \mathrm{~ms}$ period. After the controller turned on the results are obtained as given in Figure 8. The left column shows the power level variation and the corresponding penetration as well as electron temperature calculation. On the right column of the same figure, Power vs. Electron temperature plot is given for the three set of experiments.

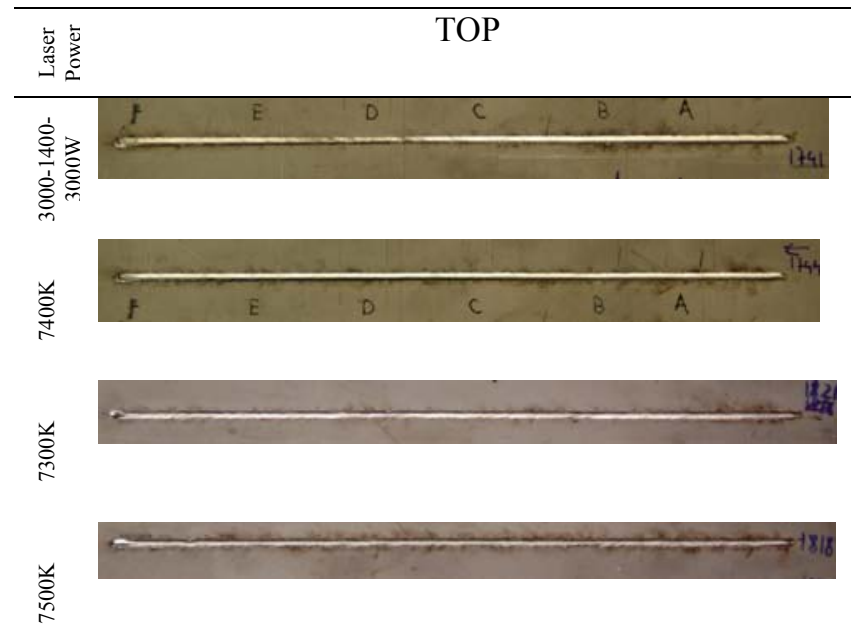

Figure 9 Top and bottom surfaces of weld seam produced by Nd:YAG laser at $60 \mathrm{~mm} / \mathrm{s}$ and $1500 \mathrm{~W}$ laser power (image width $20 \mathrm{~mm}$ ) Top surface is from characterisation experiment

Figure 9 shows the top surface look of the stainless steel plates after the experiments. The seam surface appearances are shiny and there are no oxidation observed for each seam.

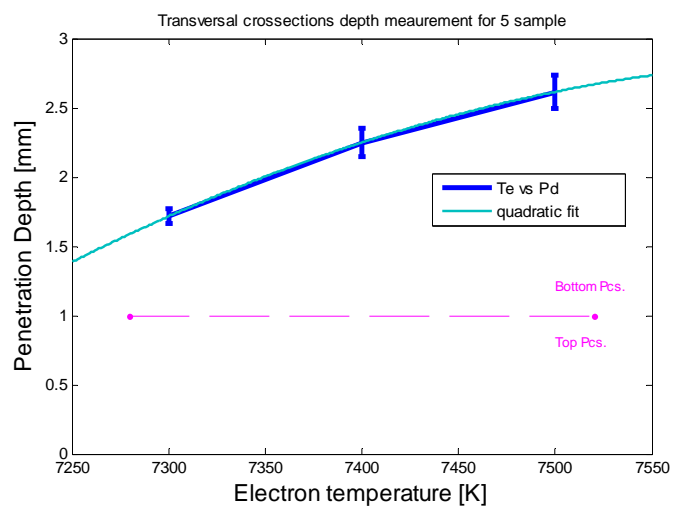

Figure 10 Transverse cross sections of Nd:Yag laser welds.
Figure 10 shows the Penetration depth variation for three different set points of the controller. $7300 \mathrm{~K}$, $7400 \mathrm{~K}$ and $7500 \mathrm{~K}$ electron temperature set point values correspond to three different penetration depths. Based on these values, a second order curve is fitted to obtain the relation.

\section{Characterization of $\mathrm{CO}_{2}$ Laser welding}

Parallel investigations have been performed with a $\mathrm{CO}_{2}$ laser source. A $2 \mathrm{~mm}$ penetration depth was chosen as the desired value, corresponding to the full penetration of the upper sheet (1-mm-thick) and half penetration of the bottom sheet (2-mm-thick). In fact, a partial penetration depth is often required for most overlap joint industrial applications where the weld must not be visible from the bottom size of the product. On the other hand the welded joint has not to be too shallow otherwise the mechanical properties of the welding product could be affected.

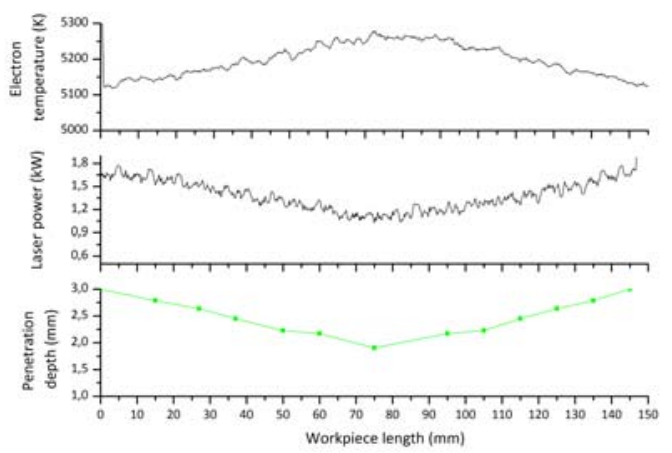

Figure $11 \mathrm{Fe}(\mathrm{I})$ electron temperature signal behaviour calculated with 421.91-517.09 nm couple of emission lines, in Ar atmosphere with variable laser power (1.7-1.2-1.7 $\mathrm{kW}$ power ramp).

The optimal $\mathrm{CO}_{2}$ laser power value, providing in our experimental conditions a 2-mm deep lap-joint, has been found to be $1.2 \mathrm{~kW}$ at a travel speed of 50 $\mathrm{mm} / \mathrm{s}$. A full penetration over the $3 \mathrm{~mm}$ thick samples was achieved for $1.7 \mathrm{~kW}$ incident laser power, for the same speed. In order to test the response of the sensor upon a dynamic change of the process conditions we ramped down and up the laser power from $1.7 \mathrm{~kW}$ to $1.2 \mathrm{~kW}$ within the same weld seam. The electron temperature has been calculated in real-time measuring the intensities of selected Fe (I) spectral lines. Figure 11 shows the Fe (I) electron temperature signal behavior and the corresponding value of the penetration depth evaluated by metallographic 
analyses of the welded joint cross sections. In case of $\mathrm{CO}_{2}$ laser welding processes, as far as the laser power and the penetration depth increase, a decrease of the electron temperature signal was measured. This temperature decrease is only apparent and has to be ascribed to the position of the light collecting system. In fact, the position of the collimator is fixed during the experiments and it does not collect the light coaxially with the laser beam but rather it is pointing towards the keyhole entrance at an angle of about $45^{\circ}$. As a consequence, being supposed that the plasma plume has temperature gradients inside its volume, as far as the keyhole gets deeper for higher incident power levels, the hottest core of the lasergenerated plasma plume goes deeper inside the keyhole. As a result, the light collected by the collimator on the keyhole surface, belongs mainly to the optical emission of the external and colder shell of the plasma plume. Therefore, an apparently lower plasma electron temperature value is measured by our system for higher incident laser powers and penetration depths. This relationship between electron temperature and penetration depth has been used to implement the closed-loop control.

For this combination of the process parameters, the values of the controller parameters $K_{p}$ and $T_{i}$ have been optimized by performing several preliminary tests and by looking for sudden and stable conditions of the controller performances. As previously explained, there is an inverse proportionality between the laser power and the electron temperature signals that results in a negative value of the $\mathrm{Kp}$ value. The controller parameter values are set to -2 and 150 for $\mathrm{Kp}$ and $\mathrm{T}_{\mathrm{i}}$, respectively for controlled experiments

\section{Controlled experiments for $\mathrm{CO}_{2}$ laser welding}

Several tests have been made to see the response of the controller settings for $\mathrm{CO}_{2}$ welding. As a result of the characterization experiments $1200 \mathrm{~W}$ is expected to be the settling value of laser power producing the desired penetration depth. The corresponding electron temperature set-point has been found to be $5500 \mathrm{~K}$ and has been kept for all of the experiments. The number of samples to average is set to 1 for the early trials. The electron temperature signal is directly used as a controller input. Figure 12 shows the laser power ouput signal for a welding test performed with an initial power level corresponding to the set point value.

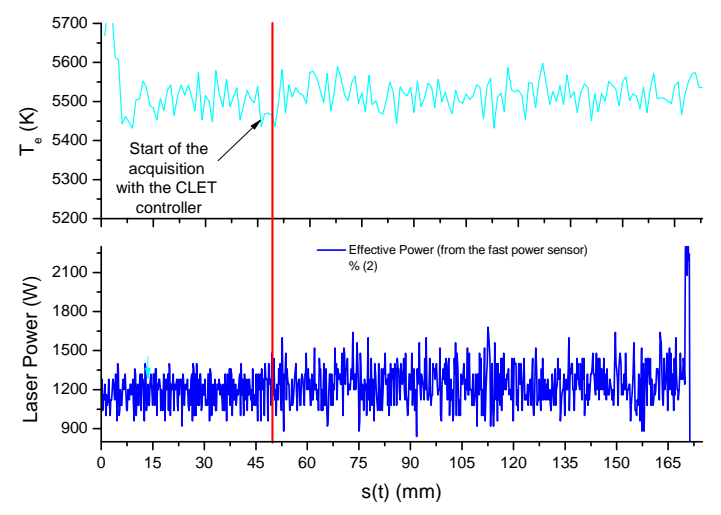

Figure 12 Controller testing on $\mathrm{CO}_{2}$ with sample to average is set to 1 . Initial power is $1200 \mathrm{~W}$

The transverse cross sections (figure 13) of the related joint exhibit an average penetration depth of $2.1 \mathrm{~mm}$. The penetration depth during welding is kept steady as in Figure 14.

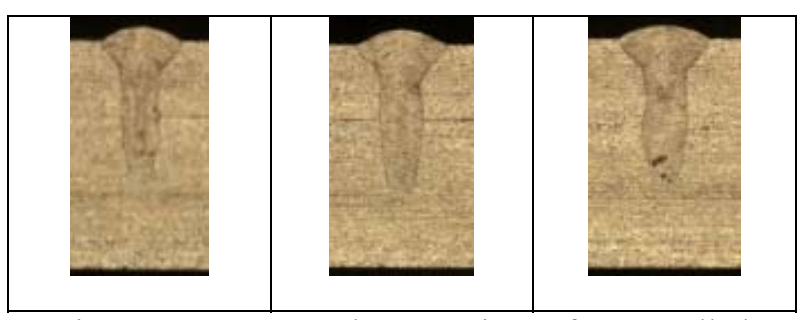

Figure 13 Transversal crossections of a controlled weld (figure 11)

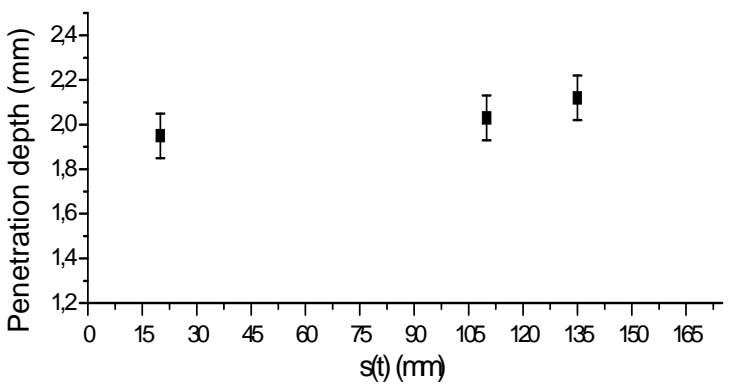

Figure 14 Penetration depth measurement (weld shown in figure 11,12)

The oscillations of the electron temperature signal are later reduced by averaging the signal. A sliding window of 10 samples in used to make the input control signal more reliable. Therefore a better result is obtained as depicted in Figure 15. 


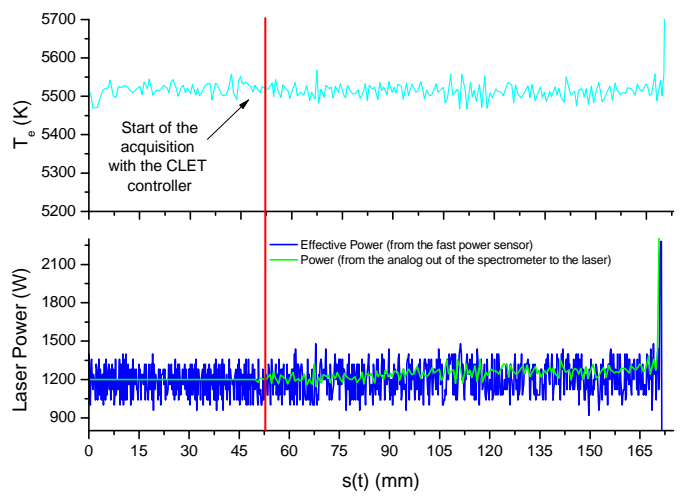

Figure 15 Controller testing on $\mathrm{CO}_{2}$ with sample to average is set to 10 . Initial power is $1200 \mathrm{~W}$

Four transverse cross section measurement proves the stability of the penetration depth as a result of averaging.

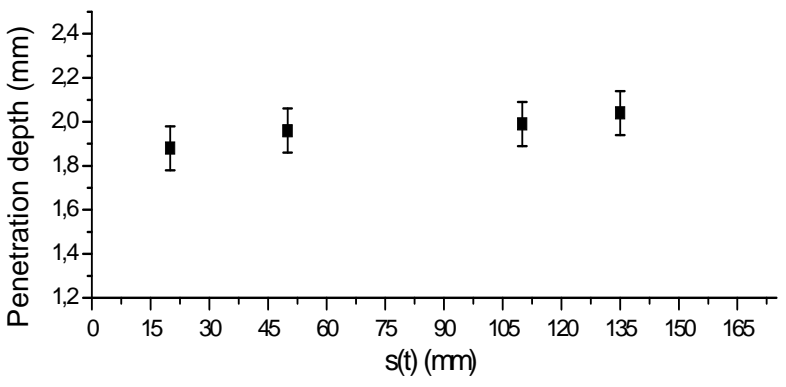

Figure 16 Penetration depth measurement on $\mathrm{CO}_{2}$ with sample to average is set to 10

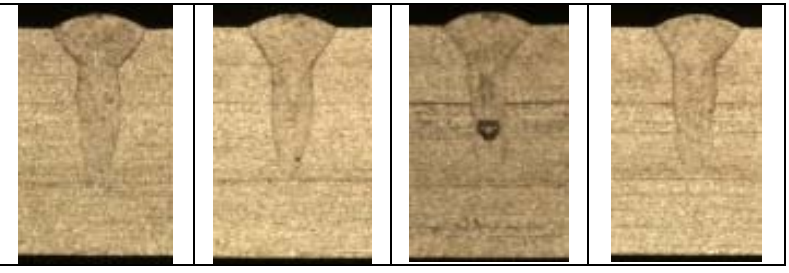

Figure 17 Transversal cross sections for average is set to 10

Next experiment is done selecting a lower incident power as an initial value, to prove the ability of the controller to settle the target penetration depth when it is switched on. For a lower incident power an apparently higher electron temperature is observed, as previously explained. Figure 18 shows how the controller brings the penetration depth and the laser power to its target value when the closed loop is activated. Cross sections in Figure 19 show the 2.1 $\mathrm{mm}$ penetration depth as the desired value attained in previous two experiments.

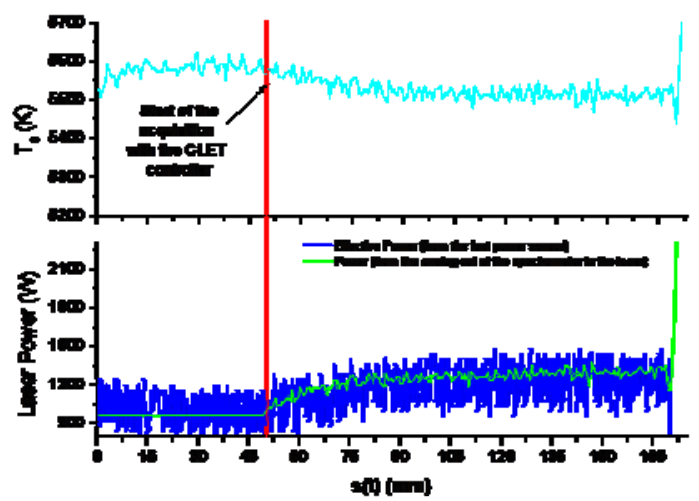

Figure 18 Controller testing on $\mathrm{CO}_{2}$ with sample to average is set to 10 starting with low incident power of $960 \mathrm{~W}$

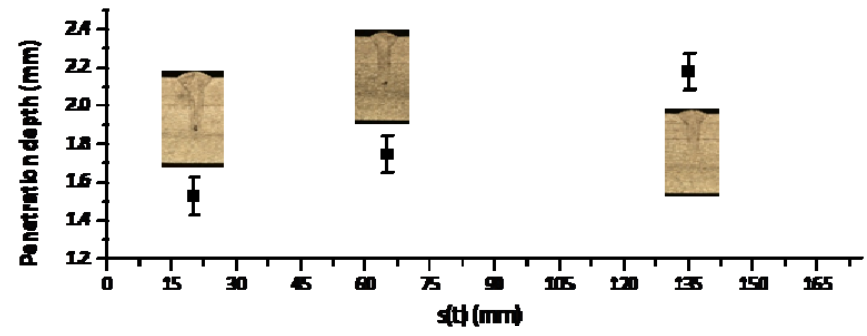

Figure 19 Penetration depth measurement and macrographs for low incident power

As a last trial the initial laser power is set to $1440 \mathrm{~W}$, $40 \%$ higher than the target value of $1200 \mathrm{~W}$ (figure 20).

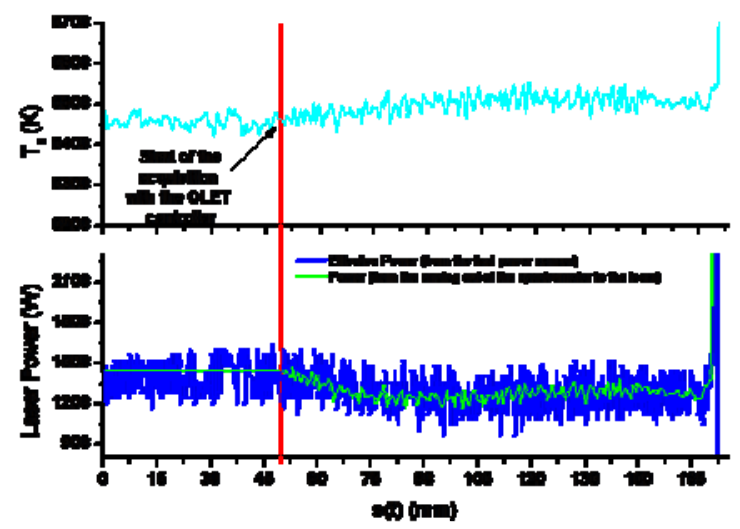

Figure 20 Controller testing on $\mathrm{CO}_{2}$ with sample to average is set to 10 starting with high incident power of $1440 \mathrm{~W}$ 
As a consequence, a higher penetration is observed in the initial part of the joint. After the controller is turned on the penetration depth is brought to the desired $2.1 \mathrm{~mm}$ value (figure21).

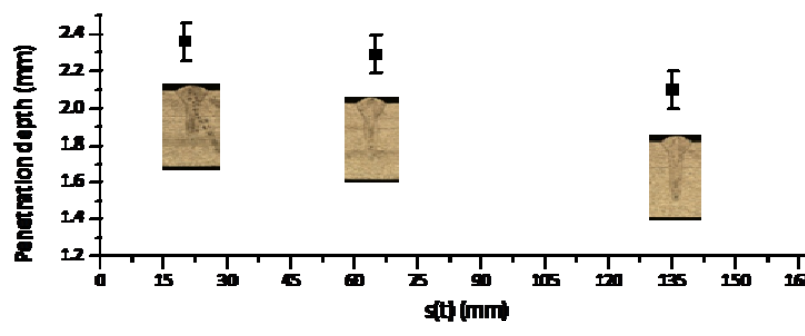

Figure 21 Penetration depth measurement and macrographs for high incident power

\section{Conclusions}

Real-time spectral measurements are utilized successfully for real-time penetration depth control of $\mathrm{Nd}$ :YAG and $\mathrm{CO}_{2}$ laser welding of AISI304 lap joints.

In particular it has been found that $\mathrm{Nd}$ :YAG penetration depth is proportional with the electron temperature calculated with $459.23 \mathrm{~nm}-495.69 \mathrm{~nm}$ CrI spectral lines. However, characterisation of the $\mathrm{CO}_{2}$ laser showed inversed relation with electron temperature calculated starting from the $\mathrm{Fe}(\mathrm{I})$ 421.91nm-517.09nm couple. A possible explanation could be that as far as the keyhole becomes deeper, as a consequence of the higher power, the plasma plume above the work piece surface goes deeper into the keyhole itself along its axial direction. Under these conditions, the light emitted by the hottest core of the plasma plume is not anymore collected by the collimator, whose angle of view remains fixed during the experiments and mainly points onto the region above the keyhole. The light detected by the sensor comes mainly from the external and colder shell of the plasma plume and an apparently lower electron temperature is measured.

Based on the characteristics of electron temperature vs. penetration depth relation the control operation is best handled by a PI type controller. The process does not require the derivative action to be in control as the high noise would make the control difficult. The noise in the process needed to be suppressed by the controller. The PI controller is also chosen to filter the signal and on the other hand to control welding process.
Characterisation prior to the calibration of the control parameters is always necessary before starting penetration controller. The characterisation determines the PI controller parameters and the relationship of the electron temperature to penetration depth.

Once the controller was calibrated for each specific experimental configuration, it was proved to adjust the laser power in order to obtain the targeted penetration depth, even when starting with a different initial laser power.

Despite the fact that several improvements can be implemented to increase its reliability, robustness of the spectrometric penetration depth controller is considered to be applicable for industrial application.

\section{References}

[1] Saludes Rodil S., Arnanz Gómez R,, M. Bernárdez J., Luis J. Miguel R., R. Perán J (2010) Laser welding defects detection in automotive industry based on radiation and spectroscopical measurements, The International Journal of Advanced Manufacturing, 133-145

[2] Ancona A., Spagnolo V., LugarÃ P. M., Ferrara M., (2001) Optical Sensor for real-time Monitoring of $\mathrm{CO}_{2}$ Laser Welding Process, In: Appl. Opt., 40 pp. $6019-6025$

[3] Zhang E.Y., (2008) Real-time weld process monitoring, Woodhead Publishing in Materials, CRC, Cambridge

[4] Contag K., Karszewski M., Stewen C., Giesen A. and Hugel H. (1999) Theoretical modelling and experimental investigations of the diode-pumped thin-disk Yb : YAG laser, Quantum Electronics, 697.

[5] Ahmed, N. (2005) New Developments in Advanced Welding. Woodhead Publishing, Cambridge.

[6] Duley, W.W. (1999) Laser Welding, John Wiley and Sons, Inc.

[7] Sibillano T., Ancona A., Berardi V. \& Lugarà P.M. (2009), A Real-Time Spectroscopic Sensor for Monitoring Laser Welding Processes, Sensors, 33763385.

[8] Konuk A.R., Aarts R.G.K.M., Huis in 't Veld A.J., Sibillano T., Rizzi D., Ancona A., Process Control of Stainless Steel Laser Welding using an Optical Spectroscopic Sensor, LiM 2011 
[9] National Institute of Standards and Technology database

[10]Konuk A.R., Aarts R.G.K.M., Huis in 't Veld A.J., Spectra analysis of the process emission during laser welding of AISI 304 stainless steel with disk and Nd:YAG laser, pp. 666-675 in Proceeding of the ICALEO 2009, Orlando, FL, USA, November, 2-5, 2009.

[11] Sibillano T., Ancona A., Rizzi D., Saludes Rodil S., Rodríguez Nieto J., Konuk A.R., Aarts R., A.J. Huis in 't Veld (2010), Study on the correlation between plasma electron temperature and penetration depth in laser welding processes. In: Phys. Proc. 5 (2010), 429-439.

\section{Acknowledgements}

This research is carried out under the contract number FP7-SME-2007-1-222279 in the seventhframe work EU project CLET.

Authors would like to acknowledge Mr. (MSc) Wei Ya for his assistance in microscopy analyses. The help of Dr. Pathiraj on discussions and manuscript is appreciated.

\section{Meet the Authors}

Ali Riza Konuk is a $\mathrm{PhD}$ researcher working at Mechanical Automation group of the University of Twente, Netherlands. He holds a M.Sc. in mechatronics and his main interests lie in the fields of system and control engineering, robotics and laser welding technology.

Ronald Aarts is associate professor in the Mechanical Automation group of the University of Twente, Netherlands. He is working on process and motion control for robotized laser welding.

Prof. Bert Huis in ' $t$ Veld is a full professor, leading the Chair of Applied Laser Technology of the University of Twente, and also works at TNO (the Netherlands Organisation for Applied Scientific Research) in Eindhoven, in the Netherlands. 